\title{
Motor Imagery Training for Gait Rehabilitation of People With Post-Stroke Hemiparesis: Practical Applications and Protocols
}

\author{
Ayelet Dunsky ${ }^{1} \&$ Ruth Dickstein ${ }^{2}$ \\ ${ }^{1}$ The Academic College at Wingate, Israel \\ ${ }^{2}$ Department of Physical Therapy, Faculty of Social Welfare and Health Sciences, Haifa University, Israel \\ Correspondence: Ayelet Dunsky, The Academic College at Wingate, Israel. Tel: 972-9-863-9308. E-mail: \\ ayelet@wincol.ac.il
}

Received: August 2, 2018 Accepted: September 24, 2018 Online Published: October 13, 2018

doi:10.5539/gjhs.v10n11p66

URL: https://doi.org/10.5539/gjhs.v10n11p66

\begin{abstract}
Over the last two decades, the use of motor imagery (MI) for post-stroke rehabilitation has significantly increased. Previous findings support the feasibility of the incorporation of specific MI exercises to improve walking skills in individuals with post-stroke hemiparesis. However, detailed practical applications and specific protocols for the implementation of MI are scarce. The objective of this manuscript is to propose practical applications for a structured MI regimen, including detailed protocols of a six-week intervention targeting gait improvement following stroke. The proposed regimen is based on previous experience with MI rehabilitation programs for gait improvement following stroke, motor learning principles with applications for stroke rehabilitation, and the PETTLEP model. The proposed detailed protocols were found to be adjusted for gait improvement of post-stroke survivors as described in several studies, and may address the targets of different rehabilitation programs. Based on motor learning principles and guidelines, an example of verbal instructions for each treatment session during six weeks of intervention is proposed. The potential of this training program to augment and extend the rehabilitation process was proven in several studies. The variety of possibilities of scenes to image allows the clinician to target specific impaired performance and disabilities. By using the proposed structure and protocols, a large number of therapists may be able to address these targets.
\end{abstract}

Keywords: motor imagery protocols, post-stroke rehabilitation, gait

\section{Introduction}

Many people with hemiparesis following stroke exhibit impaired gait performance, which is characterized by slower gait velocity and spatio-temporal asymmetry, compared with that in healthy adults (Hsu, Tang, \& Jan, 2003; Lee, Song, Lee, \& Lee, 2011). These characteristics lead to a major cause of disability, limiting the ability to function in daily activities and affecting quality of life (Hsu et al., 2003). Consequently, improving gait performance is considered to be an important goal in most rehabilitation programs (Hancock, Shepstone, Rowe, Myint, \& Pomeroy, 2017; Kumar, Chakrapani, \& Kedambadi, 2016; Lee et al., 2001). The majority of therapies address this goal by using physical repetitions, which are thought to improve motor activity by acting as stimulations for the brain to use the circuitry that facilitates voluntary movement (Hancock et al., 2017; Riberio, Silva, Costa, Cavalcanti, \& Lindquist, 2017). One weakness of this method is that recovery is dependent on the actual performance of an impaired limb (Stevens \& Stoykov, 2003).

In the last two decades a new, non-active technique based on mental practice with motor imagery (MI) has been found to enhance cortical plasticity, and has become an effective option to replace or supplement the standard physical therapy (Hanakawa, 2016; Hetu et al., 2013). MI is a term used for describing an active-cognitive process of rehearsal of imagined motor acts in the absence of overt physical movements, aimed at improving physical execution (de Vries et al., 2013; Dickstein \& Deutsch, 2007; Jackson, Lafleur, Malouin, Richards, \& Doyon, 2001; Lotze \& Halsband, 2006; Malouin \& Richards, 2010; Mulder, 2007). Engagement in MI has been shown to result in activation of overlapping regions in the brain, such as those activated while performing an actual motor task (Jeannerod, 1994; Lafleur et al., 2002; Mulder, 2007).

MI is known to be a learning process by which actions are programmed as in normal movements, but are inhibited from being executed (Malouin, Jackson, \& Richards, 2013). Thus, movement being carried out in the 
central nervous system provides 'practice' without body movement (Mulder, 2007). Consequently, it has been found to be effective in learning a new task, and in relearning and improving the performance of a learned skill, in both healthy and unhealthy populations (Dickstein \& Deutsch, 2007; Lotze \& Halsband, 2006; Malouin, Jackson, \& Richards, 2013; Schuster et al., 2011).

Based on its potential for relearning skills, MI has become a target for many researchers in the field of neurology and especially in stroke rehabilitation, at all stages (Braun, Beurskens, Borm, Schack, \& Wade, 2006; Dickstein \& Deutsch, 2007; Guerra, Lucchetti, \& Lucchetti, 2017; Jackson et al., 2001; Kho, Liu, \& Chung, 2014; Malouin, Jackson, \& Richards, 2013). Using MI interventions in treatment has been manipulated to engage motivation, cognitive processes, motor control and sensory feedback-based learning mechanisms $(\mathrm{Li}, \mathrm{Li}, \mathrm{Tan}, \mathrm{Chen}, \& \mathrm{Lin}$, 2017). However, a detailed structure of protocols that have been used during MI sessions, and/or the guiding principles of this structure, are scarce. Specific guidelines are of vital importance for clinicians who plan to implement MI interventions to their practice. Thus, the purpose of the current article is to propose guiding principles and detailed structure of the protocols for MI for gait rehabilitation following stroke.

\section{Methods}

In the proposed regimen, the program was built to progress gradually in order to meet two main rehabilitation objectives: (1) to facilitate motion of the affected extremity during walk by focusing on specific weaknesses: push-off during stance and the single-support time; and (2) to enhance practical gait within the environment, while improving speed and symmetry between the legs. As no general consensus exists regarding an MI regimen (Schuster et al., 2011), the proposed regimen was based on: previous experience with MI rehabilitation programs for gait improvement following stroke (Dickstein, Dunsky, \& Marcovitz, 2004; Dunsky, Dickstein, Ariav, Deutsch, \& Marcovitz, 2006; Dunsky, Dickstein, Marcovitz, Levy, \& Deutsch, 2008), motor learning principles with applications for stroke rehabilitation (Krakauer, 2006; Shepherd, 2001), and the PETTLEP model (Holmes \& Collins, 2006).

In the proposed rehabilitation program, each session is comprised of: (1) muscle relaxation (1-2 minutes); (2) explaining task features and environmental conditions (1-2 minutes) (Boyd \& Winstein, 2003); (3) imaging walking using a visual perspective (5-8 minutes) (Hall, Rodgers, \& Barr, 1990); (4) imaging walking using a kinesthetic perspective (5-8 minutes) (Hall et al., 1990); and (5) refocusing of awareness on the immediate environment and on body position ( 1 minute).

The muscle relaxation phase should include contraction and relaxation of different parts of the body, and practicing deep breathing - see verbal instructions in box 1 below.

\section{Box 1. Relaxation phase}

Close your eyes ... exhale and inhale slowly and deeply.

Contract your upper body and upper extremities muscles ( 3 seconds). Now relax.

Contract your lower body and lower extremities muscles ( 3 seconds). Now relax.

Contract all the muscles in your body ( 3 seconds). Now relax.

Try to stay relaxed like this, until the end of this session.

The MI training phases of the proposed program are based on the implementation of the PETTLEP framework (Holmes \& Collins, 2006) and motor learning principles.

Holmes and Collins presented the PETTLEP framework, aimed to help the designing of MI interventions for athletes; it comprises seven components: physical, environment, task, timing, learning, emotion and perspective. In the following parts, examples for the implementation of each component of the PETTLEP framework and other motor learning principles are presented. In addition, detailed descriptions of the contents of the MI training for each week of the intervention are provided in Appendices 1-5 (please note that in the example, the right leg is the affected limb):

Physical - This component describes the physical position of the individual, who in the current example presented in the current proposed program is sitting in a reclined position with eyes closed.

Environment - This component describes the environment that has to be imagined. In the current example, the environment gradually changes from a safe and isolated environment (see example in Table 1 - week 1) to a 
more complicated one with scenes crowded with furniture/people (see in Table 1 - weeks 5-6).

Task - This component describes the tasks that should be practiced during training. As mentioned earlier, the target for the current rehabilitation program is focused on two tasks: enhanced push-off and longer single support time of the affected limb. Accordingly, these two tasks appear in each of the training sessions, and are practiced repeatedly (see example in Table 1, weeks 1-4). In addition, for better retention of the tasks, exercises are applied randomly rather than in blocks, thus both tasks are practiced randomly in-between the other tasks (Krakauer, 2006) (see examples in Appendices 1-5).

Variety of tasks and environments - Improved learning occurs when participants practice a variety of associated tasks and receive feedback sporadically, in order to allow time to put together sensory cues into movement (Boyd \& Winstein, 2003). Motor learning is also dependent on the form and amount of practice (Kleim \& Jones, 2008). In order to fulfill this requirement, in the proposed program the tasks change from concentrating on the unaffected limb to concentrating on the affected leg, and then concentrating on both limbs (see example in Table 1 , week 2), and from focusing on improving push-off to enlarge the single support time on the affected limb (from week 2 to week 3 ).

Timing - This component describes the duration of the imagery. In the current example, 15-20 minutes treatment sessions are performed three times a week for six weeks. This schedule was chosen based on previous reports about improvement of gait following rehabilitation programs lasting between four to eight weeks, and because considerable enhancement has been established following three weeks of MI rehearsal (Dickstein et al., 2004; Page, Levine, Sisto, \& Johnston, 2001).

Learning - This component describes the learning or changes involved during imagery; the content of each MI session should be accommodated and modified during treatment in accordance with changes in actual gait functioning. In the current example, in order to address the changes in walking velocity the cadence of walking is increased by changing the metronome's tempo (see example in Table 1, week 4).

Problem solving - As improved learning was found to occur when the nervous system is encouraged to find active solutions to motor control problems (Kaelin-Lang, 2004; Muller \& Sternad, 2004), it is suggested that training should focus on task-specific problem solving. This can be done by asking the participant to imagine a room with a variety of furniture, and then asking him/her to find the best way to get to specific targets (see example in Table 1, week 6).

Emotion - This component describes the emotions that are related to the task. In order to engage motivation as well as emotional sensations (Holmes \& Collins, 2006; Schuster et al., 2011), reinforcement should be guided through imagery of feelings of assurance in walking and of positive achievement of the tasks. Accordingly, the instructor inspires feelings of security, serenity and satisfaction during the imagery walking (see the example of verbal instructions in Box 2 below).

Box 2. Verbal instructions for confidence and satisfaction

Watch your movements; you are very calm have confidence in yourself. You look forward with no worries and continue walking.

Feel how your back is erect, how you feel assurance equally in both feet.

Focus on the feeling of your body, your back is straight, you have confidence in yourself.

Perspective - This component describes imagery perspective. In order to address different perspectives during training, in the proposed protocols internal as well as external imagery scenes are applied (see Table 1 - example for external imagery in weeks 1 and 2, and for internal imagery in weeks 3 and 4). In addition, for better engagement in MI training as well as for better rehabilitation outcomes, different sources of sensory information should be integrated (Driver \& Spence, 2000; Kleim \& Jones, 2008). Thus, in the proposed protocols both visual and kinesthetic sensory modalities are being used. It has been shown that both kinesthetic and visual imagery are beneficial (Dickstein \& Deutsch, 2007), and a combination of these two methods is believed to lead to superior performance, and to contribute differently than if used individually, during motor learning processes (Dickstein \& Deutsch, 2007; Guillot et al., 2009; Jeannerod, 1994; Lotze \& Halsband, 2006; Malouin \& Richards, 2010; Mulder, 2007; Vergeer \& Roberts, 2006). Visual MI takes either a first- or a third-person perspective, that refers to the scenes of the participant in his/her environment (Guillot \& Collet, 2005). The participant imagines viewing himself/herself performing an action or viewing someone else performing an action (Guillot et al., 2009) 
(see example for verbal instruction in Table 1, weeks 1-2). Kinesthetic MI concentrates on somato-sensory sensations when imagining performing the action (Guillot \& Collet, 2005). The participant imagines himself/herself performing an action while sensing bodily sensations (Guillot et al., 2009) (see example for verbal instructions in Table 1, weeks 3-4).

The refocusing phase should include awareness on the actual environment and on the immediate body position see verbal instructions in Box 3 below.

Box 3. Refocusing phase

Now return to the feeling of your body here and now. Move your arms and fingers slowly.

Feel your thighs on the chair. Move your legs and toes slowly.

Feel your trunk against the chair. Move your whole body slightly on the chair.

Now, gradually return your focus to the sounds in the room. Follow my count from 5 to 1: On 1 open your eyes.

$5, \ldots 4, \ldots 3, \ldots 2, \ldots 1$.

\section{Results}

Based on the above principles and guidelines, six protocols were built. An example of detailed verbal instructions for each treatment session during five weeks of intervention can be seen on appendices 1-5 (in addition, instructions for the $4^{\text {th }}$ week can be seen at Dunsky et al., 2008 - APPENDIX 2). In addition, a summary of those appendices is presented on Table 1.

Table 1. Examples of verbal guidance in accordance with each week's targets

\begin{tabular}{|c|c|c|}
\hline Week & Target & Verbal guidance \\
\hline 1 & $\begin{array}{l}\text { Familiarization with MI gait } \\
\text { practice in an isolated place, on } \\
\text { a flat road with the focus of } \\
\text { attention placed on the timed } \\
\text { application of propulsive force } \\
\text { during push-off. }\end{array}$ & $\begin{array}{l}\text { Picture yourself walking at a comfortable pace on a path with no obstacles. } \\
\text { Look more closely at your limbs, now concentrate on your feet. Focus on each } \\
\text { foot at a time. } \\
\text { Look at your right foot. Watch how it accomplishes exactly the same } \\
\text { movements your left foot just did. See the right heel touches the path, the } \\
\text { forefoot pushes back, the foot goes up, and the foot advances forward, and } \\
\text { touches the path. }\end{array}$ \\
\hline 2 & $\begin{array}{l}\text { Focus attention on the } \\
\text { propulsive force during } \\
\text { push-off, using a 'slow-motion' } \\
\text { technique, and increase the } \\
\text { number of repetitions. }\end{array}$ & $\begin{array}{l}\text { Now visualize this very slow: you put your foot on the path, push back, you } \\
\text { lift your heel, the foot is rised and is sent forward, and then you put again the } \\
\text { heel. } \\
\text { See it one more time (repeat the same instructions). } \\
\text { Now enlarge the view so that you can see both feet. Follow them one after the } \\
\text { other: look at the right foot touches the path, pushes back, the heel is raised, } \\
\text { while the left foot touches the path, pushes back, the heel is raised, and now } \\
\text { the right heel touches the path. Watch this performance four more times. }\end{array}$ \\
\hline 3 & $\begin{array}{l}\text { Emphasis is placed on loading } \\
\text { of the affected limb during } \\
\text { stance. }\end{array}$ & $\begin{array}{l}\text { Concentrate on your right foot very slow. Feel your foot touches the path, and } \\
\text { your body weight moves over it. Focus on this feeling -your body-weight is } \\
\text { supported by your right foot, the left leg is in the air, you are stable. Now feel } \\
\text { that your left foot is sent forward, while your right foot pushes the ground, the } \\
\text { foot rises and is sent ahead. }\end{array}$ \\
\hline 4 & $\begin{array}{l}\text { Practicing previous elements } \\
\text { within the walking cycle; } \\
\text { practicing increased cadence, } \\
\text { while keeping symmetry. }\end{array}$ & $\begin{array}{l}\text { Focus on the feeling in both feet. First the right foot - touches the ground and } \\
\text { pushes backwards, while the left foot touches the ground, pushes backwards, } \\
\text { and is sent far forward. Feel this consequence four more times (the instructor } \\
\text { waits few seconds). } \\
\text { I will activate the tempo of the metronome; try to adjust yourself to this tempo. } \\
\text { Now, imagine yourself walking faster. I shall play a faster tempo. Concentrate } \\
\text { on the feelings of walking with confidence. }\end{array}$ \\
\hline
\end{tabular}




$\begin{array}{ll}5 \quad \begin{array}{l}\text { Practice gait towards significant } \\ \text { objects within the participant's } \\ \text { house. }\end{array} & \begin{array}{l}\text { See yourself in your home. You are alone, but there is few furniture that may } \\ \text { affect your path. } \\ \text { Visualize a vivid image of yourself standing near a sofa and looking at your } \\ \text { kitchen. You are erect, and relaxed. } \\ \text { You start walking at a calm pace. }\end{array} \\ & \begin{array}{l}\text { Now focus on the appearance of your feet, performing the sequence of } \\ \text { walking, one following the other. }\end{array} \\ & \begin{array}{ll}\text { Imagine the views in the street, other people that are walking on the sidewalk } \\ \text { neactice gait towards significant the cars on the road. Nothing disturbs your walk. } \\ \text { objects, on different surfaces } \\ \text { (i.e. roads or grass). }\end{array} \\ & \begin{array}{l}\text { Focus on the feelings of your feet. Adjust your steps to the surrounding. If } \\ \text { there is something in the way, walk around it, with confidence. }\end{array}\end{array}$

\section{Discussion and Conclusions}

Improving walking ability among people after a stroke is considered as a critical goal of rehabilitation (Braun et al., 2006; Dunsky et al., 2006; Dunsky et al., 2008). The possibility of a MI practice to supplement the rehabilitation process was proven in several studies (Braun et al., 2006; de Vries et al., 2013; Dickstein et al., 2004; Dickstein \& Deutsch, 2007; Dunsky et al., 2006; Dunsky et al., 2008; Guerra et al., 2017; Kho et al., 2014; Kumar et al., 2016; Lee et al., 2011; Li et al., 2017; Malouin \& Richards, 2010; Mulder, 2007; Stevens \& Stoykov, 2003).

In the current suggestion for training protocol, walking speed was chosen as the most important target for improvement as slow walking speed is considered a crucial functional limitation for people following stroke (Dickstein et al., 2004; Dickstein \& Deutsch, 2007; Dunsky et al., 2006; Dunsky et al., 2008; Hancock et al., 2017; Hsu et al., 2003). For gait speed improvement, the protocols concentrate on increasing both cadence and stride length. Training sessions are gradually developed over the intervention while reinforcing a symmetrical gait by enhancement of push-off, and by lengthening single support time on the affected foot.

Findings of studies that used the suggested MI protocols indicate increased walking speed, that may be the result of the use of metronome in the program (Dickstein et al., 2004; Dunsky et al., 2006; Dunsky et al., 2008).

The described MI practice protocols may be suggested as a practical home exercise program based solely on imagery. The improvements achieved by people who were involved in such interventions are promising and call for further research.

In summary, there is a general agreement of the use of MI practice as a rehabilitation instrument for impaired gait following stroke. In the presented protocols, the PETTLEP framework and several motor learning principles were incorporated to enable achieving the rehabilitation's targets. The variety of possibilities of scenes to image allows the clinician to target specific impaired performance and disabilities. By using the proposed structure and verbal guidance suggested, a large number of therapists may be able to address those targets.

\section{Competing Interests Statement}

The authors declare that there are no competing or potential conflicts of interest.

\section{References}

Boyd, L. A., \& Winstein, C. J. (2003). Impact of explicit information on implicit motor-sequence learning following middle cerebral artery stroke. Physical Therapy, 83, 976-989. https://doi.org/10.1093/ptj/83.11.976

Braun, S. M., Beurskens, A. J., Borm, P. J., Schack, T., \& Wade, D. T. (2006). The effects of mental practice in stroke rehabilitation: A systematic review. Archives of Physical Medicine and Rehabilitation, 87, 842-852. https://doi.org/10.1016/j.apmr.2006.02.034

de Vries, S., Tepper, M., Feenstra, W., Oosterveld, H., Boonstra, A. M., \& Otten, B. (2013). Motor imagery ability in stroke patients: The relationship between implicit and explicit motor imagery measures. Frontiers in Human Neuroscience, 7, 790. https://doi.org/10.3389/fnhum.2013.00790

Dickstein, R., \& Deutsch, J. E. (2007). Motor imagery in physical therapist practice. Physical Therapy, 87, 942-953. https://doi.org/10.2522/ptj.20060331

Dickstein, R., Dunsky, A., \& Marcovitz, E. (2004). Motor imagery for gait rehabilitation in post-stroke 
hemiparesis. Physical Therapy, 84, 1167-1177. https://doi.org/10.1093/ptj/84.12.1167

Driver, J., \& Spence, C. (2000). Multisensory perception: Beyond modularity and convergence. Current Biology, 10, R731-R735. https://doi.org/10.1016/S0960-9822(00)00740-5

Dunsky, A., Dickstein, R., Ariav, C., Deutsch, J., \& Marcovitz, E. (2006). Motor imagery practice in gait rehabilitation of chronic post-stroke hemiparesis: four case studies. International Journal of Rehabilitation Research, 29, 351-356. https://doi.org/10.1097/MRR.0b013e328010f559

Dunsky, A., Dickstein, R., Marcovitz, E., Levy, S., \& Deutsch, J. (2008). Home-based motor imagery training for gait rehabilitation of people with chronic poststroke hemiparesis. Archives of Physical Medicine and Rehabilitation, 89, 1580-1558. https://doi.org/10.1016/j.apmr.2007.12.039

Guerra, Z. F., Lucchetti, A. L. G., \& Lucchetti, G. (2017). Motor imagery training after stroke: A systematic review and meta-analysis of randomized controlled trials. Journal of Neurologic Physical Therapy, 41, 205-214.https://doi.org/10.1097/NPT.0000000000000200

Guillot, A., \& Collet, C. (2005). Contribution from neurophysiological and psychological methods to the study of motor imagery. Brain Research Reviews, 50, 387-397. https://doi.org/10.1016/j.brainresrev.2005.09.004

Guillot, A., Collet, C., Nguyen, V. A., Malouin F., Richards, C., \& Doyon, J. (2009). Brain activity during visual versus kinesthetic imagery: An FMRI study. Human Brain Mapping, 30, 2157-2172.https://doi.org/10.1002/hbm.20658

Hall, C., Rodgers, W., \& Barr, K. (1990). The use of imagery by athletes in selected sports. Sport Psychology, 4, 1-10.http://doi.org/10.1123/tsp.4.1.1

Hanakawa, T. (2016). Organizing motor imageries. Neuroscience Research, 104, 56-63. https://doi.org/10.1016/j.neures.2015.11.003

Hancock, N. J., Shepstone, L., Rowe, P., Myint, P. K., \& Pomeroy, V. M. (2017). Towards upright pedalling to drive recovery in people who cannot walk in the first weeks after stroke: Movement patterns and measurement. Physiotherapy, 103, 400-406. https://doi.org/10.1016/j.physio.2016.10.392

Hetu, S., Grégoire, M., Saimpont, A., Coll, M. P., Eugène, F., Michon, P. E., ... Jackson, P. (2013). The neural network of motor imagery: An ALE meta-analysis. Neuroscience and Biobehavioral Revews, 37, 930-949. https://doi.org/10.1016/j.neubiorev.2013.03.017

Holmes, P. S., \& Collins, D. J. (2016). The PETTLEP approach to motor imagery: A functional equivalence model for sport psychologists. Journal of Applied Sport Psychology, 13, 60-83. https://doi.org/10.1080/10413200109339004

Hsu, A. L., Tang, P. F., \& Jan, M. H. (2003). Analysis of impairments influencing gait velocity and asymmetry of hemiplegic patients after mild to moderate stroke. Archives of Physical Medicine and Rehabilitation, 84, 1185-1193. https://doi.org/10.1016/S0003-9993(03)00030-3

Jackson, P. L., Lafleur, M. F., Malouin, F., Richards, C., \& Doyon, J. (2001). Potential role of mental practice using motor imagery in neurologic rehabilitation. Archives of Physical Medicine and Rehabilitation, 82, 1133-1141. https://doi.org/10.1053/apmr.2001.24286

Jeannerod, M. (1994). The representing brain: Neural correlates of motor intention and imagery. The Behavioral and Brain Sciences, 17, 187-202. https://doi.org/10.1017/S0140525X00034026

Kaelin-Lang, A. (2004). Role of voluntary drive in encoding an elementary motor memory. Journal of Neurophysiology, 93, 1099-1103. https://doi.org/10.1152/jn.00143.2004

Kho, A. Y., Liu, K. P. Y., \& Chung, R. C. K. (2014). Meta-analysis on the effect of mental imagery on motor recovery of the hemiplegic upper extremity function. Australian Occupational Therapy Journal, 61, 38-48. https://doi.org/10.1111/1440-1630.12084

Kleim, J. A., \& Jones, T. A. (2008). Principles of experience-dependent neural plasticity: Implications for rehabilitation after brain damage. Journal of Speech, Language, and Hearing Research, 5, S225-S239. https://doi.org/10.1044/1092-4388(2008/018)

Krakauer, J. W. (2006). Motor learning: its relevance to stroke recovery and neurorehabilitation. Current Opinion in Neurology, 19, 84-90. https://doi.org/10.1097/01.wco.0000200544.29915.cc

Kumar, V. K., Chakrapani, M., \& Kedambadi, R. (2016). Motor imagery training on muscle strength and gait performance in ambulant stroke subjects - A randomized clinical trial. Journal of Clinical Diagnostic 
Research, 10, 2-5. https://doi.org/10.7860/JCDR/2016/16254.7358

Lafleur, M. F., Jackson, P. L., Malouin, F., Richards, C. L., Evans, A. C., \& Doyon, J. (2002). Motor learning produces parallel dynamic functional changes during the execution and imagination of sequential foot movements. Neuroimage, 16, 142-157. https://doi.org/10.1006/nimg.2001.1048

Lee, G., Song, C., Lee, Y., \& Lee, S. (2011). Effects of motor imagery training on gait ability of patients with chronic stroke. Journal of Physical Therapy Science, 23, 197-200. https://doi.org/10.1589/jpts.23.197

Lotze, M., \& Halsband, U. (2006). Motor imagery. Journal of Physiology, Paris, 99, $386-395$. https://doi.org/10.1016/j.jphysparis.2006.03.012

Malouin, F., \& Richards, C. L. (2010). Mental practice for relearning locomotor skills. Physical Therapy, 90, 240-251. https://doi.org/10.2522/ptj.20090029

Malouin, F., Jackson, P. L., \& Richards, C. L. (2013). Towards the integration of mental practice in rehabilitation programs. A critical review. Frontiers in Human Neuroscience, $7,576$. https://doi.org/10.3389/fnhum.2013.00576

Mulder, T. (2007). Motor imagery and action observation: Cognitive tools for rehabilitation. Journal of Neural Transmission, 114, 1265-1278. https://doi.org/10.1007/s00702-007-0763-z

Muller, H., \& Sternad, D. (2004). Decomposition of variability in the execution of goal-oriented tasks: Three components of skill improvement. Journal of Experimental Psychology. Hum Perception and Performance, 30, 212-233. https://doi.org/10.1037/0096-1523.30.1.212

Page, S. J., Levine, P., Sisto, S., \& Johnston, M. V. (2001). A randomized efficacy and feasibility study of imagery in acute stroke. Clinical Rehabilitation, 15, 233-240. https://doi.org/10.1191/026921501672063235

Schuster, C., Hilfiker, R., Amft, O., Scheidhauer, A., Andrews, B., Butler, J., ... Ettlin, T. (2011). Best practice for motor imagery: A systematic literature review on motor imagery training elements in five different disciplines. BMC Medicine, 9, 75. https://doi.org/10.1186/1741-7015-9-75.

Shepherd, R. B. (2001). Exercise and training to optimize functional motor performance in stroke: Driving neural reorganization? Neural Plasticity, 8, 121-129. https://doi.org/10.1155/NP.2001.121

Stevens, J. A., \& Stoykov, M. E. P. (2003). Using motor imagery in the rehabilitation of hemiparesis. Archives of Physical Medicine and Rehabilitation, 84, 1090-1092. https://doi.org/10.1016/S0003-9993(03)00042-X

Vergeer, I., \& Roberts, J. (2006). Movement and stretching imagery during flexibility training. Journal of Sports Sciences, 24, 197-208. https://doi.org/10.1080/02640410500131811 


\section{Appendix 1 - Motor Imagery Protocol* - Week 1}

Focus: Familiarization with motor imagery practice: Imagery of walking in a quiet place, on a level path, with no distractions

*In the following protocol the right limb is the paretic limb.

All texts presented in an Italic font are for the instructor, other should be read aloud towards the patient.

Part 1 - 2-3 minutes of relaxation: see instructions on Box 1

Part 2 - Visual Imagery (external motor imagery training) for 5-8 minutes:

Picture yourself in a very quiet place. It looks like a nice park with flowers and trees, the weather is wonderful, just as you like it.

In front of you, you can see a straight and clean path.

Picture yourself clearly, standing on this path.

Your trunk is straight, your face is looking forward with confidence.

Now view yourself start walking calmly on the path.

Look at your whole body, it is straight and relaxed.

Now, focus closely at your limbs, and gradually concentrate on your feet. Focus on each foot at a time. Begin with your left foot*.

See the heel touches the path, see how the forefoot pushes backward, and how the whole foot goes up and forward to touch the path again.

Now watch your right foot*. See how it accomplishes exactly the same movements your left foot just did, watch it slowly. See the right heel touches the path, the forefoot touches the path and pushes back while the heel goes up, see the foot goes up advances forward to touches the path again.

Watch four more steps in the same performance (the instructor should wait for 5 seconds, without instructions aloud).

Part 3 - Now see how you go a little faster, you are doing it calmly, while both feet perform the same movements (the instructor should wait for 5 seconds).

Part 4 - Now enlarge the view to see your whole body. Your walking is comfortable and relaxed. Your look is fearless, while you are watching the surrounding environment. See yourself walking like this while both limbs continue to perform the same movements smoothly.

Part 5 - Slowly, you decrease your speed, until you get to the end of the path.

You wait there for few second. See how your body is straight and relaxed, see how confident you look, exactly the way you looked before the stroke.

Now let us concentrate on the feelings inside your body while you walk.

Part 6 - Kinesthetic (internal) imagery for 5-8 minutes:

Imagine you are "getting inside" your body, while standing. Feel each parts of the body.

Feel the sensation in your back, it is erect. Feel how your weight is supported by your feet evenly. Now, turn around and watch the place you started your walking. You are planning your way to go back.

Now start walking. Feel the movements of your feet.

Feel that you are walking at your natural pace, while sensing the movement of each foot separately, try to feel the same sensations for both feet.

Now, focus on the sensation in your left foot*: feel how it touches the path, how it pushes backwards and how it comes up and goes forward to the next step. 
Now focus on the right foot*. Try to feel the same sensations you just felt in your left foot. Feel how it touches the path, how it pushes backwards, how it goes up, goes forward and touches the path again, while the weight of your body moves on it.

Part 7 - Complete this consequence four more strides, and try to feel that your body is strong and relaxed at the same time. Look forward with confidence (the instructor should wait for 5 seconds).

Focus again on the feelings of your legs. Perform four more strides, while concentrating on your knees going up and down, in the same manner (the instructor should wait for 5 seconds).

Now try to walk faster. Sense your back - it is straight and strong, you look forward with no worries, just as you used to walk before the stroke (the instructor should wait for 5 seconds).

Part 8 - Feel how you enjoy the walk. Slow down gradually, until you reach the same place you began your walk, you see a chair and you are sitting and resting.

Part 9 - 2 minutes refocusing - see Box 3 


\section{Appendix 2 - motor imagery protocol* - Week 2}

\section{Focus: Timed application of propulsive force during push-off}

*In the following protocol the right limb is the paretic limb.

All texts presented in an Italic font are for the instructor, other should be read aloud towards the patient.

Begin with Part 1 on Appendix 1.

Continue with Part 2 on Appendix 1, and add the following part:

See your movements more slowly now: you put your right* foot on the path, the forefoot pushes down and back, the heel is lifted, the whole foot rises and is sent forward, to touch the path again.

The instructor should repeat again the same instructions once more.

Now watch both your feet. Watch them as they perform those movements one by one: the left foot touches the path, pushes backwards, and just before it is lifted - the right foot touches the path, while it starts pushing backwards the left foot is lifted and goes forward to touch the path again.

Watch those movements for few more strides (the instructor should wait for 5 seconds, without instructions aloud).

Continue with Part 3 on Appendix 1, and add the following part:

While your walking becomes faster, pay attention to the way your feet push the ground, they do it much stronger than they did when you walked slower.

Continue with Part 4 on Appendix 1, and add the following part:

See how calmly you walk, even when your pace is fast, and when your feet push the ground stronger.

Perform Part 5 on Appendix 1.

Continue with Part 6 on Appendix 1, and add the following part:

In each movement you perform, try to feel that you are pushing strongly backwards with each foot at the same amount of strength.

Now feel both feet: your left foot touches the path, starts pushing backwards, while your right foot touches the path, you feel your body weight moves on it, you push strongly backwards, while you raise your left foot and send it forward to touch the path again.

Continue with Part 7 on Appendix 1, and add the following part:

When you feel your body walks faster, feel how hard you push the ground. You still feel calm and relaxed. You feel that both your legs are evenly strong.

Perform few more steps like that (the instructor should wait for 5 seconds).

Perform Parts 8 and 9 on Appendix 1. 


\section{Appendix 3 - motor imagery protocol* - Week 3}

\section{Focus: Loading of the affected lower extremity during stance and increasing gait speed}

*In the following protocol the right limb is the paretic limb.

All texts presented in an Italic font are for the instructor, other should be read aloud towards the patient.

\section{Begin with Part 1 on Appendix 1.}

Continue with Part 2 on Appendix 1, and add the following part:

Now watch the performance of both feet, one after the other. While looking at them focus on the way you shift your body weight onto each foot, how each foot pushes the ground, and goes to the next step.

Now perform those movements very slowly. First your right* foot, see how it touches the surface, and your body weight shifts on to it. Now stay there and expand your view, and watch how your whole body is actually supported by your right leg. You look completely stable. Now see your left foot swinging as you send it far forward. Now your right foot is pushing down and back, you lift it and take it far forward to touch the path again.

The instructor should repeat the above instruction once more, and then ask the patient to perform four more steps, without the verbal instructions.

Continue with Part 3 on Appendix 1, and add the following part:

While walking faster concentrate on the view of your weight supported by your right leg and by your left leg, in the same manner.

Continue with Part 4 on Appendix 1, and add the following part:

Perform six continuous steps, and at the end of the sixth step, lift a hand (the instructor should measure the time passes until the patient lifts his/her hand-and write it down).

Perform Part 5 on Appendix 1.

Continue with Part 6 on Appendix 1, and add the following part:

Let's concentrate on your right foot again, this time do it slowly. Feel it touches the ground, feel the shifting of your body weight over the foot. Try to concentrate on this feeling - all your body weight is supported by your right leg, while your left foot is in the air. You are upright and stable. Now try to feel that the left foot is sent far forward, while your right foot pushes the ground. When the left foot touches the path again, you raise your right leg, swing it forward and touch the path again.

The instructor should repeat the above instruction once more.

Now feel the movements of both your legs, one after the other. Feel the same sensations for both. First the left foot touches the path, your body-weight shifts over it, it pushes backwards, while the right foot touches the path, now feel your body weight shifts over it, now you are slowly pushing backwards, while sending your left foot far forward to touch the path again.

Continue with Part 7 on Appendix 1, and add the following part:

While walking faster, feel each time that your whole body-weight "leans" on each foot in the same manner, in addition, try to feel that you are pushing stronger into the surface.

Perform six more steps; at the end of the sixth step raise your hand (the instructor should measure the time passes until the patient lifts his/her hand - and write it down).

Perform Parts 8 and 9 on Appendix 1. 


\section{Appendix 4 - motor imagery protocol* - Week 5}

\section{Focus: Imagery practice of walking toward meaningful targets indoors.}

*In the following protocol the right limb is the paretic limb.

For protocol of week 4 see Dunsky et al., 2008

All texts presented in an Italic font are for the instructor, other should be read aloud towards the patient.

\section{Begin with Part 1 on Appendix 1}

Continue with visual Imagery:

See yourself in a familiar place - your house or another familiar indoor environment. There are no people around, but there is some furniture that could affect the walking path.

Picture yourself standing near a sofa planning to walk to the kitchen.

As always, see at how confidence and calm you look.

Now see yourself start walking towards the kitchen in a comfortable pace.

Gradually ignore the surroundings and concentrate on the appearance of your feet.

Watch each leg separately. Begin with your left foot*.

See how it touches the floor, how it pushes backward, goes up and touches the floor again.

Now try to see it again in slow motion: the foot touches the floor, pushes back, and now see how it goes up and far, far forward before it touches the floor.

Now watch your right foot*. See how it accomplishes exactly the same movements your left foot just did. See the right heel touches the floor, the forefoot pushes very strong back while the heel goes up, see the foot advances forward, and again touches the floor.

Now look at both your feet. Watch them as they perform those movements one by one, at the exact same manner. Concentrate on the way each foot is pushing strongly backwards and then going far forward.

Perform Part 4 on Appendix 1, and add the following part:

While walking this path from the sofa to the kitchen, you are able to change the walking course if necessary, to pass by a chair or a table on your way - any of those changes does not change your comfortable walking, until you get to the kitchen.

You stop there and drink a glass of water.

Look at yourself. You are part of the surroundings. You are sure of yourself, just as you were before the stroke.

Let's concentrate on the sensations inside your body, while you will go back toward the sofa.

Perform Parts 6 and 7 on Appendix 1, and add the following part:

The whole time you walk towards the sofa, feel you have no hesitations. Feel confidence equally in both your legs.

Gradually slow down until you reach the sofa, you turn around and sit on it, and relax.

Perform Part 9 on Appendix 1. 


\section{Appendix 5 - motor imagery protocol - Week 6}

Focus (same as week 5, but outdoor): Imagery practice of walking toward meaningful targets; walking on different terrains, such as roads, and grass

*In the following protocol the right limb is the paretic limb.

All texts presented in an Italic font are for the instructor, other should be read aloud towards the patient.

\section{Begin with Part 1 on Appendix 1}

Continue with visual Imagery:

Visualize a picture of a familiar place (for example, the sidewalk outside your house), and see yourself standing there at a beginning of a path between you and a grocery shop across the street.

As always, see how confident and calm you are.

See how you start walking towards the shop.

Gradually ignore the surroundings and concentrate on the appearance of your feet.

Watch each leg separately. Begin with your left foot*.

See how it touches the sidewalk, how it pushes backward, goes up far forward, and touches the sidewalk again.

Now try to imagine your right foot* this time very slow: the foot touches the sidewalk, pushes backward, goes up and forward, and touches the sidewalk again.

The instructor should repeat the previous instruction once more.

Now, watch both feet as they perform those movements one by one, at the exact same manner. Concentrate on the way each foot is pushing strongly backwards and then going far forward.

Continue in this manner for a few more steps forward (The instructor should wait 5 seconds).

Perform Part 4 on Appendix 1, and add the following part:

While you walk towards the shop, despite the people around you, despite the changes in the environment, you are very relaxed and confident. You are able to adjust yourself to changes in the course, if necessary. Any change, in the course, does not affect your confident walking.

Try to see yourself walking six steps at the same pace. After the sixth step, raise one hand (the instructor should measure the time passes until the patient lifts his/her hand - and write it down).

The instructor can use a metronome for external tempo, and ask the patient to adjust his steps to the beats of the metronome, while performing six steps, and looking at whole his body.

Watch your movements. You look forward with no worries and continue walking with confidence. Note the sidewalk, the houses along the road, the road with the cars.

Continue in this manner for a few more steps forward, until you reach the grocery.

You now stop to rest a little.

Look around you, there are people and cars, and a pleasant breeze.

Look at yourself. You are part of the surroundings. You are erect and sure of yourself, just as you were before your stroke.

Let's concentrate on your sensations inside your body, while you will go back toward your house.

Perform Parts 6 and 7 on Appendix 1, and add the following part:

The whole time you go back towards your house, imagine the sights on the street, the cars on the road, other people that are walking on the sidewalk near you. They do not disturb your walk.

The instructor can use the metronome again and ask the patient to pace him/herself to the beats.

While walking adjust your steps to the environment, to the changes in the sidewalk. If there is something on the sidewalk, you walk around it, with no fear.

Continue in this manner for a few more steps forward, and gradually slow down until you reach the entrance of your house. There is a bench over there, and you are sitting and resting. 
Perform Part 9 on Appendix 1.

\section{Copyrights}

Copyright for this article is retained by the author(s), with first publication rights granted to the journal.

This is an open-access article distributed under the terms and conditions of the Creative Commons Attribution license (http://creativecommons.org/licenses/by/4.0/). 